\title{
Introduction to the Special Issue on Data-Enabled Discovery for Industrial Cyber-Physical Systems
}

\author{
Raju Gottumukkala ${ }^{1} \cdot$ Peter Beling ${ }^{2}$ \\ Published online: 29 December 2020 \\ (C) Springer Nature Switzerland AG 2020
}

The technological revolution enabled by the Industry 4.0 revolution is fusing the physical and digital worlds through the confluence of various technologies (i.e., Internet of Things (IoT), Artificial Intelligence, Cyber-physical Systems, and smart factories) [1] [2]. Industrial systems in several domains ranging from manufacturing, transportation, energy, defense, automotive, and buildings operate in an environment that is highly dynamic, safety-critical, and uncertain [3]. Bringing automation and connectivity into these industrial systems introduces system complexity inherent to systemlevel integration and operation. It is our endeavor to engineer future industrial systems that not only augment automation technologies, but are also safe and dependable.

Cyber-physical systems are engineered systems built from seamless integration of computation (i.e., sensing, computing, and networking) and physical components. There are many data-centric challenges related to the implementation, operation, control, and optimization of these systems with a high degree of complexity. These challenges arise from data, model, or system-level integration [4]. Data related issues arise from inherent nature of sensory data, that includes noisy, uncertain, partially informative, and dynamic data. The modeling-related challenges range from how to improve the robustness of machine learning models, primarily when used

This article belongs to the Topical Collection: Data-Enabled Discovery for Industrial Cyber-Physical Systems

Guest Editor: Raju Gottumukkala

Raju Gottumukkala

raju.gottumukkala@louisiana.edu; raju@louisiana.edu

Peter Beling

pb3a@virginia.edu

1 Department of Mechanical Engineering \& NSF Center for Visual and Decision Informatics, University of Louisiana at Lafayette, Lafayette, USA

2 Department of Engineering Systems and Environment, University of Virginia, Charlottesville, VA, USA in the place of physics-based models based on sound assumptions across many engineering domains. Finally, when machine learning models are integrated into existing industrial processes, one has to deal with performance and system-level constraints such as cost, computational time, and budget. This special issue is dedicated to presenting papers that deal with various issues about data-driven discovery with industrial cyber-physical systems. The special issue covers a broad range of topics that includes cyber-physical integration, scalability and reliability of cyber-infrastructure, and systems engineering. The application of these topics ranges broadly from power engineering to medical diagnosis to aircraft maintenance.

Physics-based models have a high degree of transparency, as the transition from inputs and outputs are clearly explained with first-principle models. On the other hand, most machine learning models estimate variables directly from data using black-box approaches. In applications where there is a need for a high degree of transparency and accuracy, due to cost or safety reasons such as health domain or industrial systems, explainable AI provides details of why the model is saying what it is saying. Krishnamurthy et al. [5] offer an explainable AI framework for images that have applications in industrial systems or medical diagnostics. Ferdowsi et al. [6] present a data-driven approach to classify power behavior dynamics on Solid State Transformers (SST) in a microgrid using machine learning. Unlike traditional physics-based techniques, machine learning methods effectively capture the nonlinear dynamics associated with the uncertainty and variability of renewable energy resources in power distribution networks.

Future industrial systems will be dependent on cyberinfrastructure for monitoring, diagnostics, and control. Integrating operations and maintenance data into common data platforms enable industrial systems to perform both predictive and prescriptive maintenance. Choubey et al. [7] provide a holistic prescriptive maintenance framework to predict impending failures to help manage unplanned downtime at optimal cost. Prescriptive maintenance provides data-driven strategies for both part replacement and repair solution 
recommendations. Cloud computing provides both scalability and reliability at an infrastructure level. Still, critical infrastructure such as power systems uses local computational infrastructure, and reliability guarantees are an essential for these systems. Henning and Hasselbring [8] present a scalable stream processing framework with an emphasis on reliability for monitoring and analysis of IoT streams in the power distribution systems.

Feature selection and engineering directly affect the machine learning algorithm's computational performance (i.e., accuracy, efficiency, cost, etc.), the model's interpretability and explainability, and cost of machine learning projects. Chomiak [9] presents a novel feature extraction method to improve image classification for computer-aided health diagnosis. The proposed method was evaluated on both binary and multi-class classification across three different datasets. These methods have potential applications in automated medical imaging systems.

Two different aspects of feature selection problems are covered in this special issue. Carson et al. [10] provide a machine learning-based approach for feature selection for aircraft safety and maintenance. These features are used to predict whether an incident will be identified during aircraft maintenance or aircraft operation and what specific type of incident will occur. One of the important elements of data-driven modeling of cyber-physical systems is quantifying the cost of data-driven methods. While accuracy is an important consideration in the deployment of machine learning methods, the cost of data acquisition, the computational cost associated with feature extraction, and the cost of misclassification are also important. Meekins et al. [11] provide a novel feature selection technique that optimizes both cost and accuracy.

\section{References}

1. K. Schwab, The fourth industrial revolution. Foreign Affairs (2015), https://www.foreignaffairs.com/articles/2015-12-12/ fourth-industrial-revolution.
2. A. Trauth-Goik, Repudiating the fourth industrial revolution discourse: a new episteme of technological Progress. World Futures 74, 1-24 (2020)

3. C. Arnold, D. Kiel, K.-I. Voigt, How the industrial internet of things changes business models in different manufacturing industries. Int. J. Innov. Manag. 20(08), 1640015 (2016)

4. N. Baker, F. Alexander, T. Bremer, A. Hagberg, Y. Kevrekidis, H. Najm, M. Parashar, A. Patra, J. Sethian, S. Wild, K. Willcox, S. Lee, Workshop report on basic research needs for scientific machine learning: core technologies for artificial intelligence $(\mathrm{N}$., United States, 2019. Web.). https://doi.org/10.2172/1478744

5. V. Krishnamurthy, K. Nezafati, E. Stayton, V. Singh, Explainable AI framework for imaging-based predictive maintenance for automotive applications and beyond. Data-Enab Discov Appl 4(1), 1$\mathrm{xx}$ (2020) [editorial staff please insert correct reference to this SI]

6. F. Ferdowsi, M. Hosseini, M. Hosseini, Classification of emergent dynamics in microgrids utilizing an entropic complexity-based feature extractor. Data-Enab Discov Appl 4(1), 1-xx (2020) [editorial staff please insert correct reference to this $\mathrm{SI}]$

7. S. Choubey, R. Benton, R. Benton, A holistic end-to-end prescriptive maintenance framework. Data-Enab Discov Appl 4(1), 1-Xx (2020) [editorial staff please insert correct reference to this SI]

8. S. Henning, W. Hasselbring, Scalable and reliable multidimensional sensor data aggregation in data streaming architectures. Data-Enab Discov Appl 4(1), 1-xx (2020) [editorial staff please insert correct reference to this $\mathrm{SI}]$

9. T. Chomiak, Recurrence quantification analysis statistics for image feature extraction and classification. Data-Enab Discov Appl 4(1), 1-xx (2020) [editorial staff please insert correct reference to this SI]

10. J. Carson, K. Hollingsworth, R. Datta, A. Segev, Failing and not falling (F\&!F): Data-enabled classification learning of aircraft accidents and incidents. Data-Enab Discov Appl 4(1), 1-xx (2020) [editorial staff please insert correct reference to this $\mathrm{SI}$ ]

11. R. Meekins, S. Adams, K. Farinholt, S. Polter, P.A. Beling, ROC with cost Pareto frontier feature selection using search methods. Data-Enab Discov Appl 4(1), 1-xx (2020) [editorial staff please insert correct reference to this $\mathrm{SI}]$

Publisher's Note Springer Nature remains neutral with regard to jurisdictional claims in published maps and institutional affiliations. 